Palabras clave/ Arqueología urbana, cartografía, materiales constructivos, Valdivia.

$\Delta$ Keywords/ Urban archaeology, mapping. building materials, Valdivia.

$\Delta$ Recepción/ 12 agosto 2015

$\Delta$ Aceptación/ 17 septiembre 2015

\title{
Carta arqueológica del área fundacional de Valdivia: arquitectura, materiales constructivos y tradiciones cerámicas.
}

\section{Archaeological map of Valdivia's foundational area: Architecture, construction materials and ceramic traditions.}

\section{Simón Urbina-Araya}

Arqueólogo, Universidad de Chile, Chile. Magister en Historia, mención Etnohistoria, Universidad de Chile, Chile. Laboratorio de Arqueología, Dirección

Museológica, Universidad Austral de Chile, Chile. simon.urbina@uach.cl

\section{Leonor Adán-Alfaro}

Arqueóloga, Universidad de Chile, Chile.

Doctora en Historia, mención Etnohistoria Universidad de Chile, Chile.

Dirección Museológica, Universidad Austral de Chile, Chile.

leonor.adanauach.cl

\section{Constanza Chamorro-Palma}

Arquitecta, Universidad Austral de Chile, Chile. constanza.chamorro.pagmail.com

RESUMEN/ Este trabajo actualiza la carta arqueológica para el área fundacional de la ciudad de Valdivia. Se presentan nuevas dataciones absolutas en distintos sectores de la ciudad que permiten discutir su correlación con las etapas y fases urbanísticas planteadas por los estudios históricos. El análisis distribucional y tipológico de ladrillos, tejas curvas y planas, cerámicas indígenas y europeas, así como evidencias funerarias, permite integrar información funcional, diacrónica y sincrónica de los yacimientos estudiados en la última década. Se valora el estudio de los materiales constructivos como un paso fundamental para la arqueología histórica y otras disciplinas interesadas en el asentamiento humano y las modificaciones del paisaje urbano en Valdivia en una perspectiva de larga duración. ABSTRACT/ This paper updates the archaeological map of the foundational area of Valdivia. New absolute dates are introduced for several urban areas which discuss their correlation with urban phases and stages as presented by historical studies. The distributional and typological analysis of bricks, curved and flat tiles, indigenous Mapuche-Huilliche and European ceramics, as well as funerary evidence permits the integration of functional, diachronic and synchronic information of the archaeological sites studied in the past decade. The study of building materials is valued as a main step towards historical archeology and other disciplines interested in human settlements and changes in the Valdivia's urban landscape from a long term perspective.

\section{EL ESTUDIO DE LOS MATERIALES CONSTRUCTIVOS ARQUEOLÓGICOS'.}

La investigación arqueológica del 'área fundacional' de Valdivia ofrece un significativo complemento a los vacios existentes en la secuencia de planos y cartografías publicadas entre los siglos XVII y XIX (Guarda 2009). En este trabajo presentamos los resultados de análisis cronológicos efectuados sobre materiales constructivos diagnósticos -tejas y ladrillos- (Urbina, Adán y Chamorro 2017), provenientes de excavaciones y recolecciones superficiales efectuadas en el radio urbano de Valdivia y alrededores (imagen 1 e imagen 2).
Durante los últimos diez años las evidencias de arquitectura arqueológica in situ y restos dispersos de elementos constructivos se han incrementado progresivamente y con ello los registros de materialidades y técnicas constructivas del período Colonial y Republicano, especialmente aquellas de la etapa industrial (ca. 1870-1960) (Guarda 2001), como fundaciones de bloques canteados de piedra laja y pilares o muros de ladrillo (imagen 3), los cuales utilizan morteros de arena y conchilla, cal y cemento (Urbina et al. 2012). Un hallazgo novedoso en el ámbito de las techumbres es el de "tejas planas con gancho y acanaladuras" características del norte de Europa durante el siglo XIX (Espinosa 1859 y Lámina 6, $n^{\circ}$ 110: 369, Touttsaint de Sens 1860) Ejemplares fragmentados de estas tejas se registran asociados a las fundaciones de la Aduana en el sitio Contraloría Regional, en estratigrafía - o bajo el subsuelo- en los sitios Plaza Pedro de Valdivia, Huerta Campus Cultural-Universidad Austral de Chile (UACh) y Casa Prochelle-1 (Isla Teja); también, a nivel superficial en el sitio Carlos Anwandter-1 o reutilizados como relleno en entretechos, como ocurre en el actual Conservatorio de Música de la UACh (imagen 4 e imagen 5). 


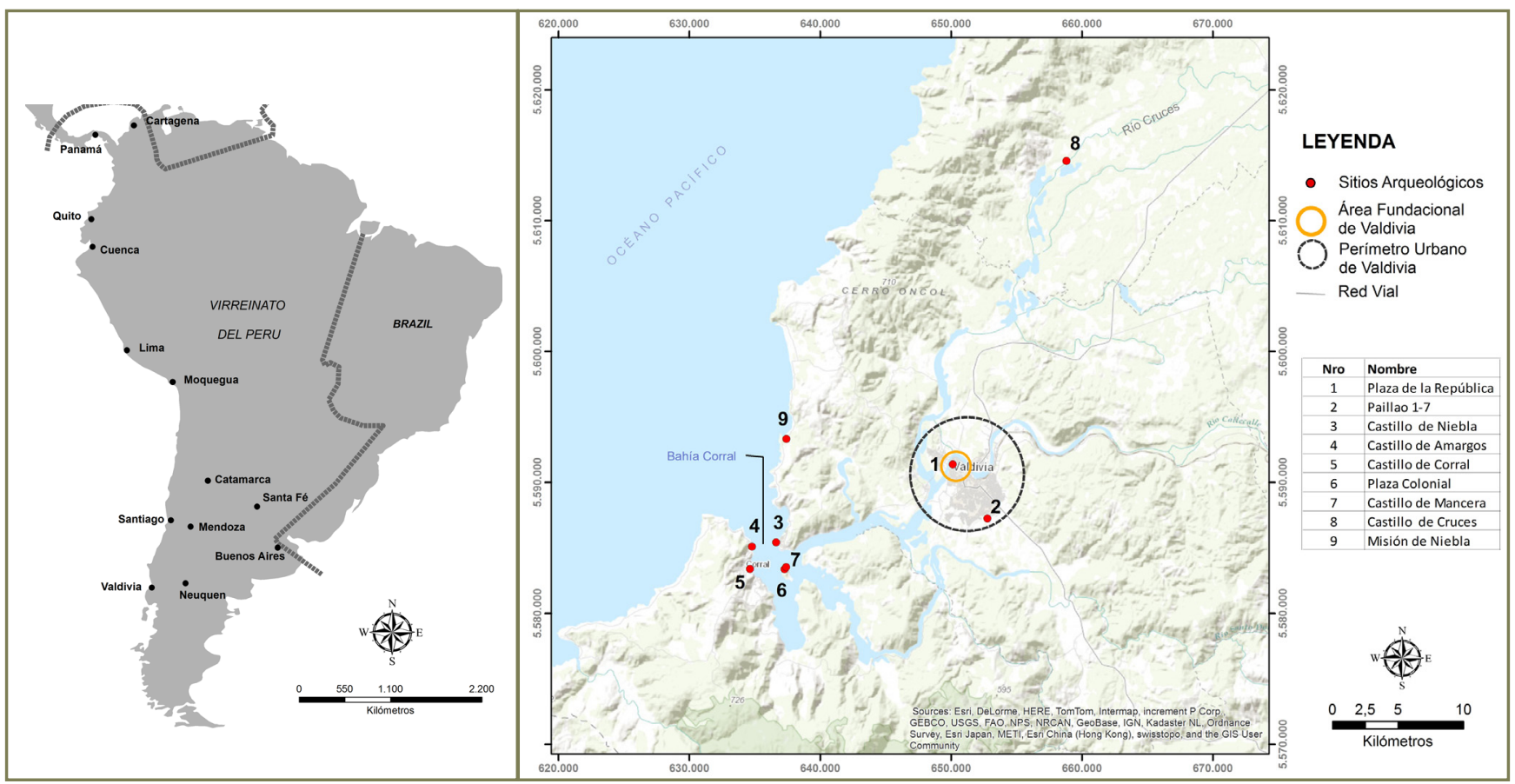

Imagen 1. Ubicación de la ciudad de Valdivia en el Virreinato del

Imagen 2. Valdivia y su sistema defensivo (fuente: Los autores).

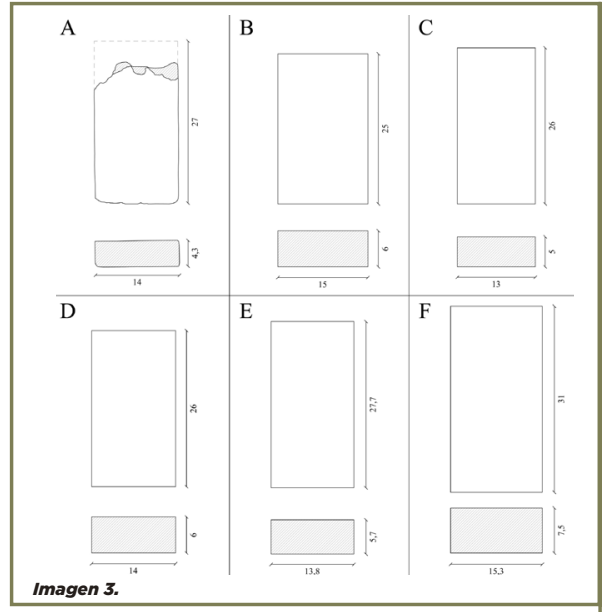

A

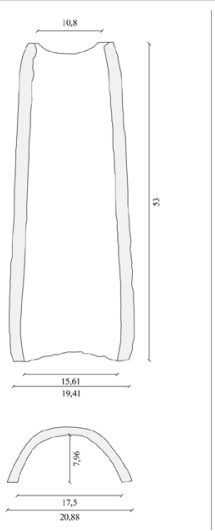

B

$+\frac{2}{c}$
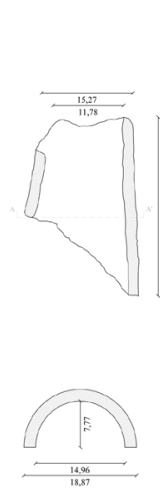

C

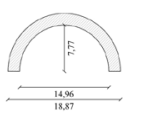

$\mathrm{C}$

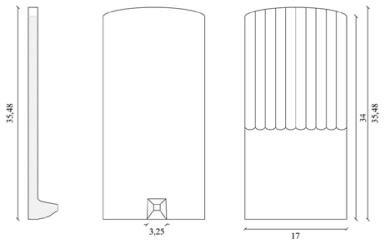

$\underset{17}{\stackrel{4.12}{\pi}}$

Imagen 4.

Imagen 3. Ladrillos arqueológicos. Modificado de Gordon 2017: 57).

BB) Contraloria Regional (sig/o XIX-XX)

$3 C$ ) Cervecerla Anwandter (siglo XIX-XX)

3D) Casino de Valdivia (sig/o XVili).

(SF) Camiglo XVI-XVII).

(fuente: (os autores).

Imagen 4. Tejas arqueo/ógicas

Sicica $($ siglo XVI), (fuente:

4B) Casa fuerte Santa Sylvia Villarrica (siglo XVI). Colección Dirección Museológica-UACh.

4C) Conservatorio de Música UACh (s. XIX-XX). (fuente: Los autores).

so musleras.

온

讨
3A) Casa fuerte Santa SyMVia, Villarrica (siglo XVI) (fuente:

Imagen 5. Perfil de fragmentos de tejas arqueológicas

Serie B1-B6. Tejas planas con gancho.
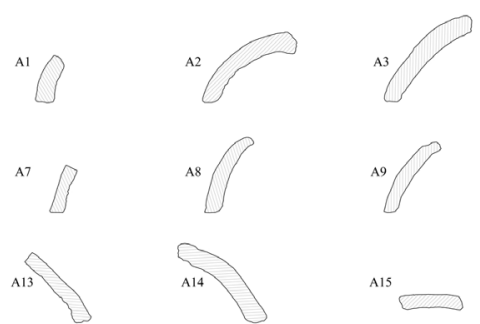

A19
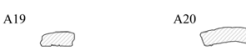

${ }^{\mathrm{A}} 25$

A26
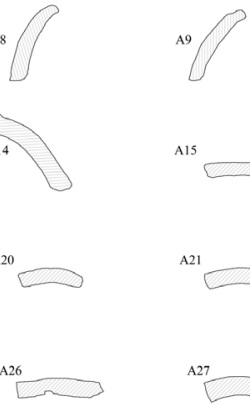

$\sqrt{8}$

A15

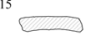

$\longrightarrow$
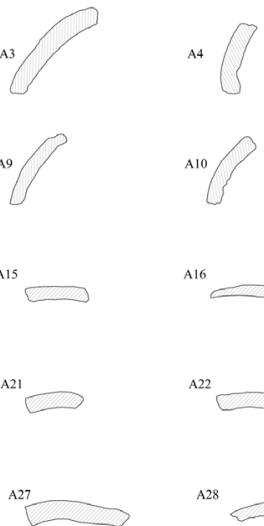

s5

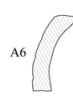

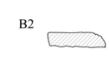

${ }^{\mathrm{B}} \mathrm{\square}$
A28

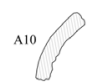

A16

A22

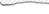

$\sim$

B4

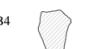

B5

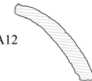

A11 7
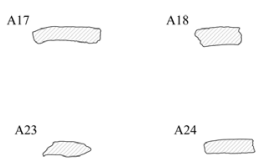

$\longrightarrow$ 


\begin{tabular}{|c|c|c|c|c|c|c|c|c|c|c|}
\hline \multirow[b]{3}{*}{ Material } & \multirow[b]{3}{*}{ Período } & \multicolumn{8}{|c|}{ Atributos } & \\
\hline & & \multicolumn{2}{|c|}{ Color } & \multicolumn{2}{|c|}{ Largo (cms) } & \multicolumn{2}{|c|}{ Ancho (cms) } & \multicolumn{2}{|c|}{ Espesor (cms) } & \\
\hline & & Carta Munsell & Nombre & Mín. & Máx. & Mín. & Máx. & Mín. & Máx. & Procedencia de las muestras (sitios) \\
\hline Ladrillo & $\begin{array}{l}\text { Colonial } \\
\text { (s. XVI-XIX) }\end{array}$ & $\begin{array}{l}\text { 10R } 4 / 6,10 R \\
5 / 6,10 R 5 / 8 \\
10 R 6 / 8\end{array}$ & Rojo/Rojo claro & 25,0 & 31,0 & 14,0 & 15,6 & 5,1 & 7,5 & $\begin{array}{l}\text { Villarrica*: Santa Sylvia y Camino } \\
\text { Pedregoso-4/Valdivia'*: Misión de Niebla, } \\
\text { Castillo Corral, Torreón El Barro y Los } \\
\text { Canelos, Casino de Valdivia. }\end{array}$ \\
\hline Ladrillo & $\begin{array}{l}\text { Republicano } \\
\text { (s. XIX-XX) }\end{array}$ & $\begin{array}{l}2.5 Y R 7 / 4,2.5 Y R \\
6 / 8,2.5 Y R 5 / 6, \\
2.5 Y R 5 / 4\end{array}$ & $\begin{array}{l}\text { Rojo/Rojo claro/ } \\
\text { Café rojizo/Café } \\
\text { rojizo claro }\end{array}$ & 25,0 & 27,0 & 12,8 & 15,3 & 5,1 & 6,6 & $\begin{array}{l}\text { Valdivia**: Plaza Pedro de Valdivia, Plazoleta } \\
\text { Acharán Arce, Mina de Vidrio-1, Cervecería } \\
\text { Anwandter, Yungay } 773 \text {, Contraloria Regional. }\end{array}$ \\
\hline $\begin{array}{l}\text { Teja curva } \\
\text { o muslera }\end{array}$ & $\begin{array}{l}\text { Colonial } \\
\text { (s. XVI-XIX) }\end{array}$ & $\begin{array}{l}10 R 5 / 8,2.5 Y R \\
6 / 8,5 Y R 6 / 8 \\
\text { 5YR } 5 / 6\end{array}$ & $\begin{array}{l}\text { Rojo/Rojo } \\
\text { claro/Rojo } \\
\text { amarillento/ } \\
\text { Amarillo rojizo }\end{array}$ & - & 53,0 & 15,3 & 19,4 & 1,1 & 2,4 & $\begin{array}{l}\text { Villarrica*: Santa Sylvia/ Valdivia**: Carlos } \\
\text { Anwandter-1, Plaza Pedro de Valdivia, Plaza } \\
\text { de la República, Independencia con Arauco, } \\
\text { Contraloría Regional, Yungay 773, Casa } \\
\text { Extensión UACh, Prorrectoría UACh, Huerta } \\
\text { - Campus Cultural UACh, Castillo de Niebla y } \\
\text { Corral, Plaza Colonial Mancera. }\end{array}$ \\
\hline $\begin{array}{l}\text { Teja plana } \\
\text { con gancho }\end{array}$ & $\begin{array}{l}\text { Republicano } \\
\text { (s. XIX-XX) }\end{array}$ & $\begin{array}{l}2.5 \mathrm{YR} 6 / 8,5 \mathrm{YR} \\
6 / 8,7.5 \mathrm{YR} 7 / 6, \\
7.5 \mathrm{YR} 8 / 6\end{array}$ & Amarillo rojizo & 35,0 & 35,5 & 16,6 & 17,3 & 1,2 & 1,8 & $\begin{array}{l}\text { Valdivia**: Contraloría Regional, Plaza Pedro } \\
\text { de Valdivia, Huerta Campus Cultural-UACh, } \\
\text { Casa Prochelle-1, Carlos Anwandter-1. }\end{array}$ \\
\hline
\end{tabular}

Tabla 1. Caracterización de los materiales constructivos procedentes de Valdivia y su jurisdicción (fuente: Los autores).

\section{ELABORACIÓN DE LA CARTA} ARQUEOLÓGICA DE VALDIVIA

Considerando las dataciones por

termoluminiscencia obtenidas para la vajilla cerámica y materiales constructivos en el perímetro urbano de la ciudad, las cuales abarcan desde el siglo XV hasta inicios del siglo XX (tabla 2$)^{3}$, en este apartado presentamos la zonificación y carta arqueológica del 'área fundacional' de Valdivia.

En la tabla 1 se sistematizan los atributos básicos -color y dimensiones- registrados en las muestras de ladrillos y tejas arqueológicas -completas o fragmentadas(imagen 6 e imagen 7), segregando aquellos ejemplares que provienen de sitios asignados al período Colonial (ca. 1552-1820) y Republicano (ca. 18201960). Sin duda, sobre este conjunto de evidencias resta un estudio detenido de las propiedades y rasgos derivados del proceso de manufactura artesanal o industrial, procedencia, preparación y calidad de las arcillas utilizadas, así como el reconocimiento del elenco de fábricas de donde proceden las piezas ${ }^{2}$

En suma, el análisis sistemático de materiales constructivos arqueológicos aportará decisivamente a la comprensión de las distintas modalidades urbanísticas e hitos fundacionales de Valdivia, dirimiendo la asignación de diversos atributos tecnológicos y tipologías constructivas a fases y períodos del área fundacional de Valdivia.
Para el análisis se ha utilizado el plano de la ciudad en formato AutoCad 2010, con curvas de nivel cada dos metros. Se han resaltado en escala de grises las cotas superiores o altas de la ciudad. En rojo transparente se ha delimitado el polígono que ocupa el área edificada del plano holandés (ca. 1643) sobrepuesto al plano actual de Valdivia, posicionando la ruka ${ }^{4}$ ubicada en la isla Teja, los barracones holandeses y el fuerte, todos con líneas de color negro. El esquema de la Plaza Real o castillo de Valdivia (ca. 1647-1798) se ha transcrito desde el plano elaborado por Antonio Birt (ca. 1763. imagen 8) escalando en metros la planta y posicionándola de acuerdo a la propuesta de Guarda (2009) Por último, se ha transcrito el plano de la línea de defensa o cerca de Duce (ca. 1785. imagen 9), escalado en metros y sobrepuesto al plano actual de Valdivia con una línea continua de color rojo. Como referencia se consideró la ubicación de los torreones El Barro y Los Canelos, actualmente in situ

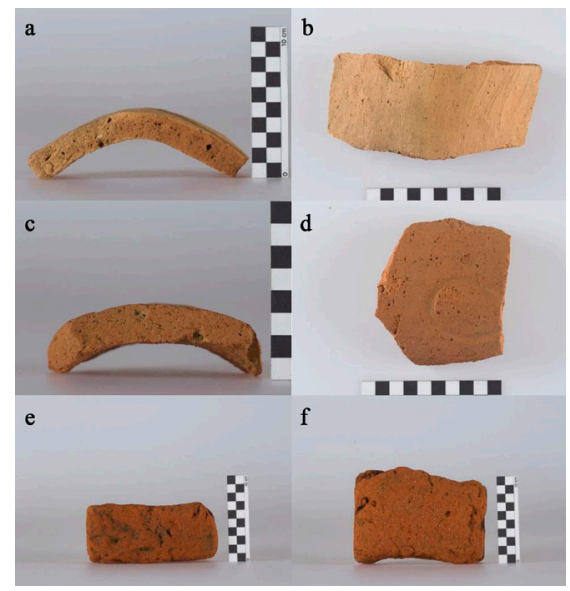

Imagen 6. Tejas y ladrillos del periodo Colonia. 6a-b: Teja curva, sitio Carampangue 1. 6c-d: Teja curva con marca, sitio Plaza de La República. Ge-f: Ladrillo, sitio La Misión de Niebla. (fuente: Los autores).

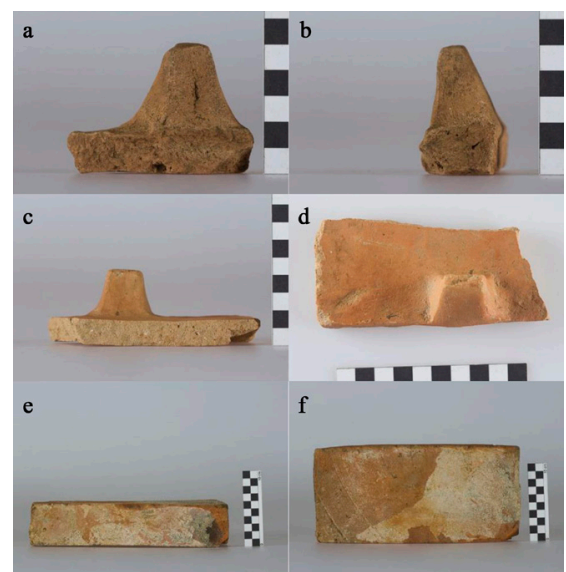

Imagen 7. Tejas y ladrillos del período Republicano. 7a-b: Teja plana con gancho, sitio Carlos Anwandter-1.
7c-d: Teja plana con gancho, sitio Contraloría Regional. 7e-f: Ladrillo, sitio Contraloría Regional. (fuente: Los autores).

2 Para información histórica respecto de las fábricas de teja y ladrillo, véase Guarda 2001: 58, 351-352, 496, 690 


\begin{tabular}{|c|c|c|c|c|c|}
\hline MUESTRA & Sitio & Estructura/Unidad/ Nivel (cms) & Material/Tipo & Edad (AP) & FECHA \\
\hline UCTL 2826 & Carlos Anwandter-1 & Unidad 1, nivel 40-50 & Cerámica sin torno con engobe rojo & $535 \pm 50$ & 1475 DC \\
\hline UCTL 2898 & Carlos Anwandter-1 & Pozo 1, nivel 58-70 & Teja curva & $450 \pm 50$ & $1565 \mathrm{DC}$ \\
\hline UCTL 1923 & Casino de Valdivia & Muro Sur (Hallazgo 5) & Ladrillo (in situ) & $405 \pm 40$ & $1600 \mathrm{DC}$ \\
\hline UCTL 2773 & Contraloría Regional & Trinchera Este & Cerámica sin torno monócroma & $390 \pm 35$ & $1620 \mathrm{DC}$ \\
\hline UCTL 2901 & Contraloría Regional & Trinchera E, nivel 170-180 & Teja curva & $385 \pm 35$ & $1630 \mathrm{DC}$ \\
\hline UCTL 2810 & Casa Prochelle-1 & Monitoreo Patio W & Cerámica Estilo Valdivia & $375 \pm 35$ & $1635 \mathrm{DC}$ \\
\hline UCTL 2931 & Independencia con Arauco & Pozo 1B, rasgo 1, nivel 0-100 & Ladrillo & $325 \pm 30$ & $1660 \mathrm{DC}$ \\
\hline UCTL 2910 & Independencia con Arauco & Pozo 1B, rasgo 1, nivel 0-100 & Teja curva & $350 \pm 40$ & $1665 \mathrm{DC}$ \\
\hline UCTL 2927 & Independencia con Arauco & Pozo 1B, rasgo 1, nivel 0-100 & Ladrillo & $345 \pm 35$ & $1670 \mathrm{DC}$ \\
\hline UCTL 2815 & Plaza de La República & Unidad 18, nivel 95-105 & Cerámica Estilo Valdivia & $335 \pm 30$ & $1675 \mathrm{DC}$ \\
\hline UCTL 2896 & Plaza de la República & Pozo 33, nivel 155-165 & Teja curva & $340 \pm 35$ & $1675 \mathrm{DC}$ \\
\hline UCTL 2897 & Plaza de la República & Pozo 1, nivel 80-90 & Teja curva & $335 \pm 35$ & $1680 \mathrm{DC}$ \\
\hline UCTL 2899 & Yungay 800 Casa Extensión UACh. & Trinchera $1 \mathrm{~A}$, nivel $60-70$ & Teja curva & $330 \pm 30$ & 1685 DC \\
\hline UCTL 2905 & Yungay 800 Casa Extensión UACh. & Trinchera 1B, nivel 30-40 & Teja curva & $320 \pm 30$ & $1695 \mathrm{DC}$ \\
\hline UCTL 2922 & Independencia con Arauco & Pozo 1A & Teja curva & $300 \pm 30$ & 1715 DC \\
\hline UCTL 2844 & Cerveceria Anwandter & Trinchera & Ladrillo & $290 \pm 30$ & $1720 \mathrm{DC}$ \\
\hline UCTL 1972 & Casino de Valdivia & Muro Norte (Hallazgo 3) & Ladrillo (in situ) & $285 \pm 30$ & $1720 \mathrm{DC}$ \\
\hline UCTL 2917 & Independencia con Arauco & Pozo 1B, rasgo 1, nivel 0-100 & Teja curva & $295 \pm 25$ & $1720 \mathrm{DC}$ \\
\hline UCTL 2936 & Yungay 800 Casa Extensión UACh. & Pozo 2B, nivel 60-70 & Ladrillo & $295 \pm 25$ & $1720 \mathrm{DC}$ \\
\hline UCTL 2924 & Mirador Yungay & Trinchera 1, bajo incendio (ca. 1909) & Teja curva & $290 \pm 30$ & $1725 \mathrm{DC}$ \\
\hline UCTL 1971 & Casino de Valdivia & Muro Norte (Hallazgo 3) & Ladrillo (in situ) & $270 \pm 25$ & $1735 \mathrm{DC}$ \\
\hline UCTL 2911 & Independencia con Arauco & Pozo 1B, rasgo 1, nivel 0-100 & Teja curva & $280 \pm 30$ & 1735 DC \\
\hline UCTL 2912 & Yungay 773 & Rec. Superficial, nivel 0-5 & Teja curva & $280 \pm 30$ & $1735 \mathrm{DC}$ \\
\hline UCTL 2918 & Plaza de la República & Pozo 33, nivel 85-95 & Teja curva & $280 \pm 25$ & 1735 DC \\
\hline UCTL 2930 & Plazoleta los Laureles & Pozo 1B, nivel 0-10 & Ladrillo & $280 \pm 25$ & $1735 \mathrm{DC}$ \\
\hline UCTL 2913 & Contraloría Regional & Muro Sector A, relleno & Teja plana & $275 \pm 25$ & $1740 \mathrm{DC}$ \\
\hline UCTL 2771 & Contraloría Regional & Trinchera Este & Mayólica Panamá Polícromo & $260 \pm 25$ & $1750 \mathrm{DC}$ \\
\hline UCTL 1973 & Casino de Valdivia & Muro Norte (Hallazgo 3) & Ladrillo (in situ) & $250 \pm 25$ & $1755 \mathrm{DC}$ \\
\hline UCTL 2921 & Costanera con Carampangue & Rec. Superficial, nivel 0-5 & Teja curva & $260 \pm 25$ & $1755 \mathrm{DC}$ \\
\hline UCTL 2916 & Prorrectoría UACh. & Rec. Superficial, nivel 0-5 & Teja curva & $250 \pm 25$ & $1765 \mathrm{DC}$ \\
\hline UCTL 2772 & Contraloría Regional & Trinchera Este & Cerámica con torno (botija) & $240 \pm 20$ & $1770 \mathrm{DC}$ \\
\hline UCTL 2909 & Casa Prochelle-1 & Rec. Superficial, nivel 0-5 & Teja plana & $240 \pm 20$ & $1775 \mathrm{DC}$ \\
\hline UCTL 2923 & Plaza Pedro de Valdivia & Rec. Superficial, nivel 0-50 & Teja curva & $225 \pm 20$ & $1790 \mathrm{DC}$ \\
\hline UCTL 2823 & Plaza de La República & Unidad 18, nivel 45-55 & Cerámica con incrustaciones & $315 \pm 30$ & 1795 DC \\
\hline UCTL 2908 & Huerta Don Juan-Campus Cultura & Rec. Superficial, nivel 0-5 & Teja curva & $220 \pm 25$ & 1795 DC \\
\hline UCTL 2904 & Huerta Don Juan-Campus Cultura & Rec. Superficial, nivel 0-5 & Teja plana & $215 \pm 20$ & $1800 \mathrm{DC}$ \\
\hline UCTL 2915 & Carlos Anwandter-1 & Rec. Superficial, nivel 0-5 & Teja curva & $195 \pm 20$ & $1820 \mathrm{DC}$ \\
\hline UCTL 2925 & Casa Prochelle-1 & Rec. Superficial, nivel 0-5 & Ladrillo & $185 \pm 15$ & $1830 \mathrm{DC}$ \\
\hline UCTL 2938 & Contraloría Regional & Excavación, bajo incendio (ca. 1909) & Ladrillo & $180 \pm 15$ & $1835 \mathrm{DC}$ \\
\hline UCTL 2935 & Plaza Pedro de Valdivia & Rec. Superficial, nivel 0-50 & Ladrillo & $175 \pm 15$ & $1840 \mathrm{DC}$ \\
\hline UCTL 2926 & Carlos Anwandter-1 & Rec. Superficial, nivel 0-5 & Ladrillo & $170 \pm 15$ & $1845 \mathrm{DC}$ \\
\hline UCTL 2939 & Cervecería Anwandter & Rec. Superficial, nivel 0-5 & Ladrillo & $155 \pm 15$ & $1860 \mathrm{DC}$ \\
\hline UCTL 2903 & Plaza Pedro de Valdivia & Rec. Superficial, nivel 0-50 & Teja plana & $140 \pm 15$ & $1875 \mathrm{DC}$ \\
\hline UCTL 2933 & Plazoleta Acharán Arce (Salesiano) & Pozo 1, nivel 50-60 & Ladrillo & $135 \pm 12$ & $1880 \mathrm{DC}$ \\
\hline UCTL 2937 & Yungay 773 & Rec. Superficial, nivel 0-5 & Ladrillo & $125 \pm 10$ & $1890 \mathrm{DC}$ \\
\hline UCTL 2914 & Carlos Anwandter-1 & Rec. Superficial, nivel 0-5 & Teja plana & $120 \pm 20$ & $1895 \mathrm{DC}$ \\
\hline UCTL 2928 & Lab. Arqueología Campus Cultural & Pozo 1, nivel 40-50 & Ladrillo & $120 \pm 12$ & 1895 DC \\
\hline UCTL 2929 & Torreón el Barro & Rec. Superficial, nivel 0-5 & Ladrillo & $105 \pm 10$ & $1910 \mathrm{DC}$ \\
\hline UCTL 2920 & Conservatorio de Música & Rec. Superficial, entre techo & Teja plana & $95 \pm 10$ & $1920 \mathrm{DC}$ \\
\hline
\end{tabular}

Tabla 2. Dataciones por termoluminiscencia para el área fundacional de Valdivia (fuente: Los autores). 
Los sitios arqueológicos han sido dotados de una simbología específica considerando la presencia y combinación de tipos de materiales constructivos y fragmentos cerámicos de tradición indígena o europea (imagen 10 e imagen 11).

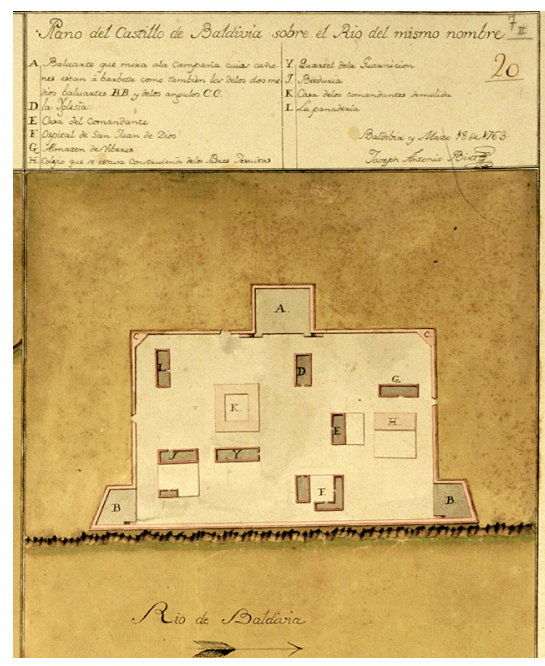

Imagen 8. Plano del castillo de Valdivia, por Antonio Birt (ca. 1763). (fuente: Ms. 400/ $n^{0} 7-11,5$ Biblioteca Nacional de Cata/uña, Barcelona).
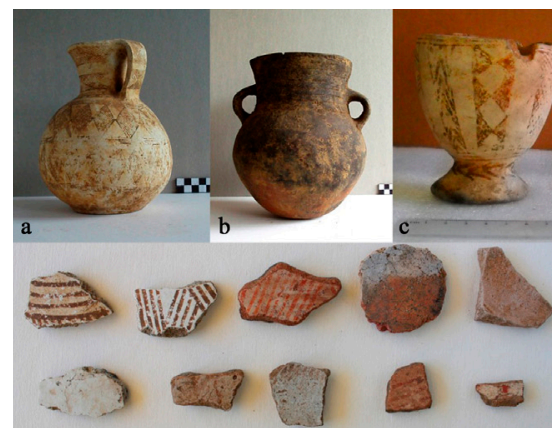

d

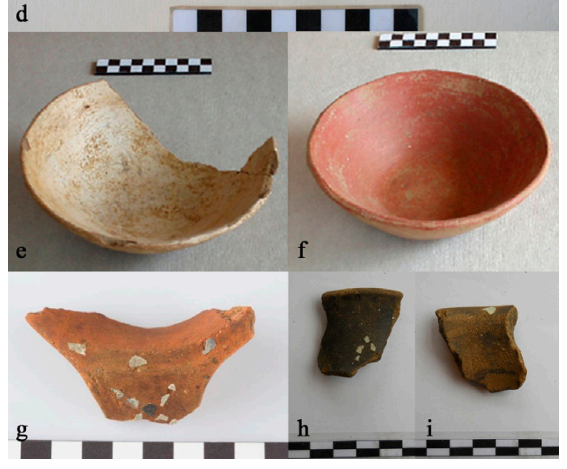

Imagen 10. Cerámica de Tradición Indígena.

10a) Jarro Estilo Valdivia, sitio Las Mulatas.

10b) Olla con estriamiento anular cuello-cuerpo, sitio Las Mulatas 10c) Copa Estilo Valdivia, sitio Millahililin 2 (comuna de Máfili). distribuidos en el área fundacional de Valdivia. 10e-f) Platos pintados, sitio Las Mulatas.

10e-f) Platos pintados, sitio Las Mulatas. Plaza de la República (fuente: Los autores).
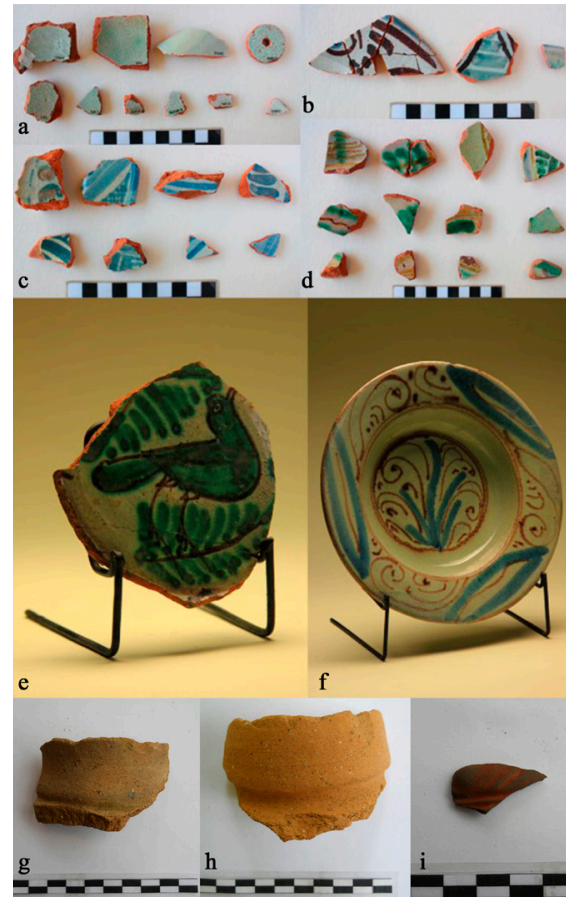

Imagen 11. Cerámica de Tradición Europea

17a) Tipo Panamá Liso

716) Tipo Panamá Policromo A.

The Pana blanco

Limas más Allä.

Palicromo A, sitio Castillo de

Cruces (comuna de Mariquina).

(comuna de Mariquina).

llg-h) Bordes de botijas, sitio Plaza de la República.

Iii) Cerámica fina incisa (búcaro), sitio Plaza de la República

(fuente: Los autores).

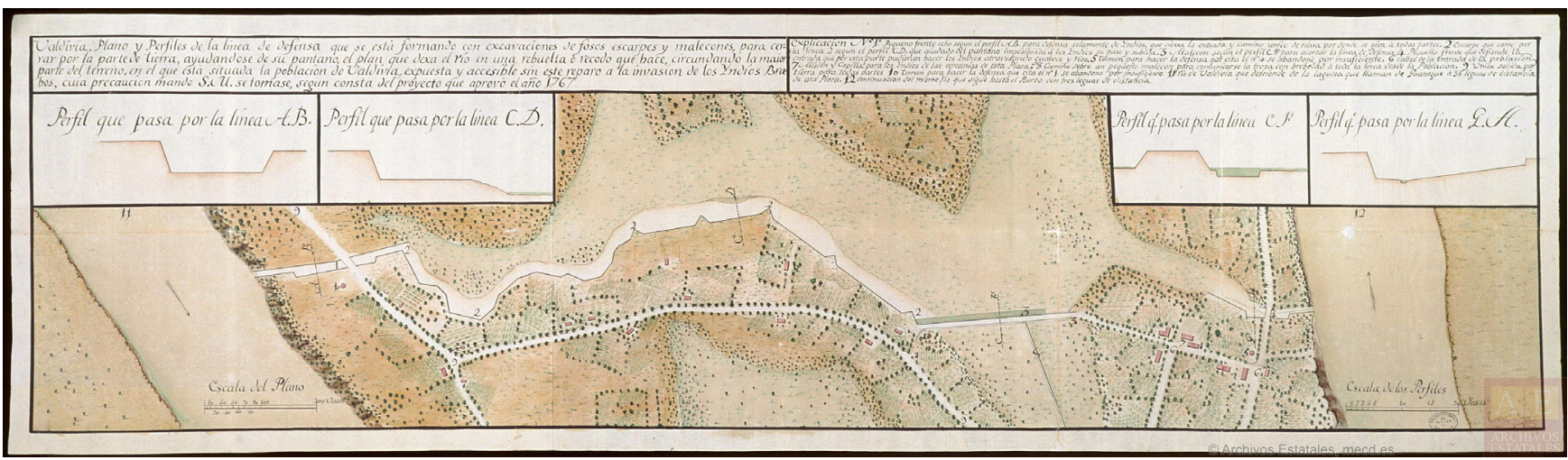

Imagen 9. "Valdivia. Plano y perfiles de la línea de defensa" (ca. 1785) (fuente: Mapas y Planos de Chile y Perú, n 83, Archivo General de Indias, Sevilla). 


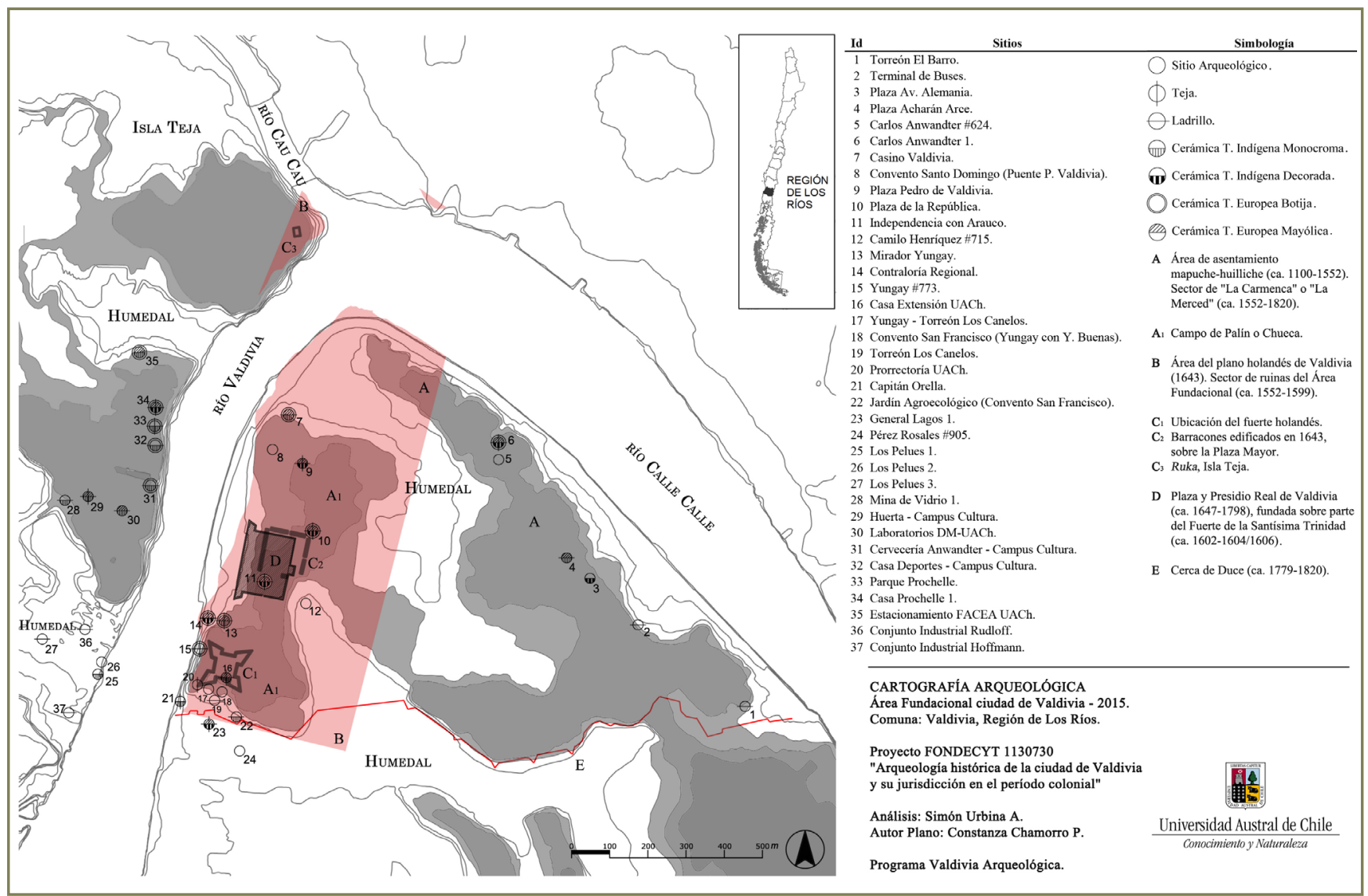

Imagen 12. Zonificación arqueológica del Área Fundacional de Valdivia al año 2016 (fuente: Los autores).

La primera lámina, denominada Zonificación Arqueológica, ubica los sitios arqueológicos conocidos sobre la topografía que caracteriza el centro histórico de la ciudad, agregando información sobre zonas que definen las distintas etapas históricas de asentamiento humano y la presencia de materiales constructivos y tipos cerámicos diagnósticos por sitio (imagen 12, ver simbología).
La Carta Arqueológica, por su parte, enfatiza la distribución de los puntos -sitios arqueológicos- sobre la planta urbana actual, conservado la zonificación arqueológica determinada por la primera planta urbana de Valdivia (imagen 13, letra B); la localización del fuerte y barracones holandeses, de la ruka consignada en el margen nororiental de la isla Teja en el plano holandés del s. XVII (imagen 13, letra C1, C2 y C3), de la Plaza Murada (imagen 13, letra D) y la cerca de Duce (imagen 13, letra E). permitiendo una comprensión integral de los recursos arqueológicos, su expresión territorial y emplazamiento en el área de confluencia del río Cau-Cau y Calle-Calle, allí donde nace el río Valdivia o Guadalafquén. El aumento de sitios o recursos arqueológicos en más de un 54\% -de 24 a 37 sitios para el 'área fundacional' de Valdivia- en menos de 3 años, responde al sostenido proceso de intervenciones generadas en las etapas de diseño de obras y ejecución de obras, paralelamente a las investigaciones científicas financiadas por fondos estatales e internos provistos por la UACh. 


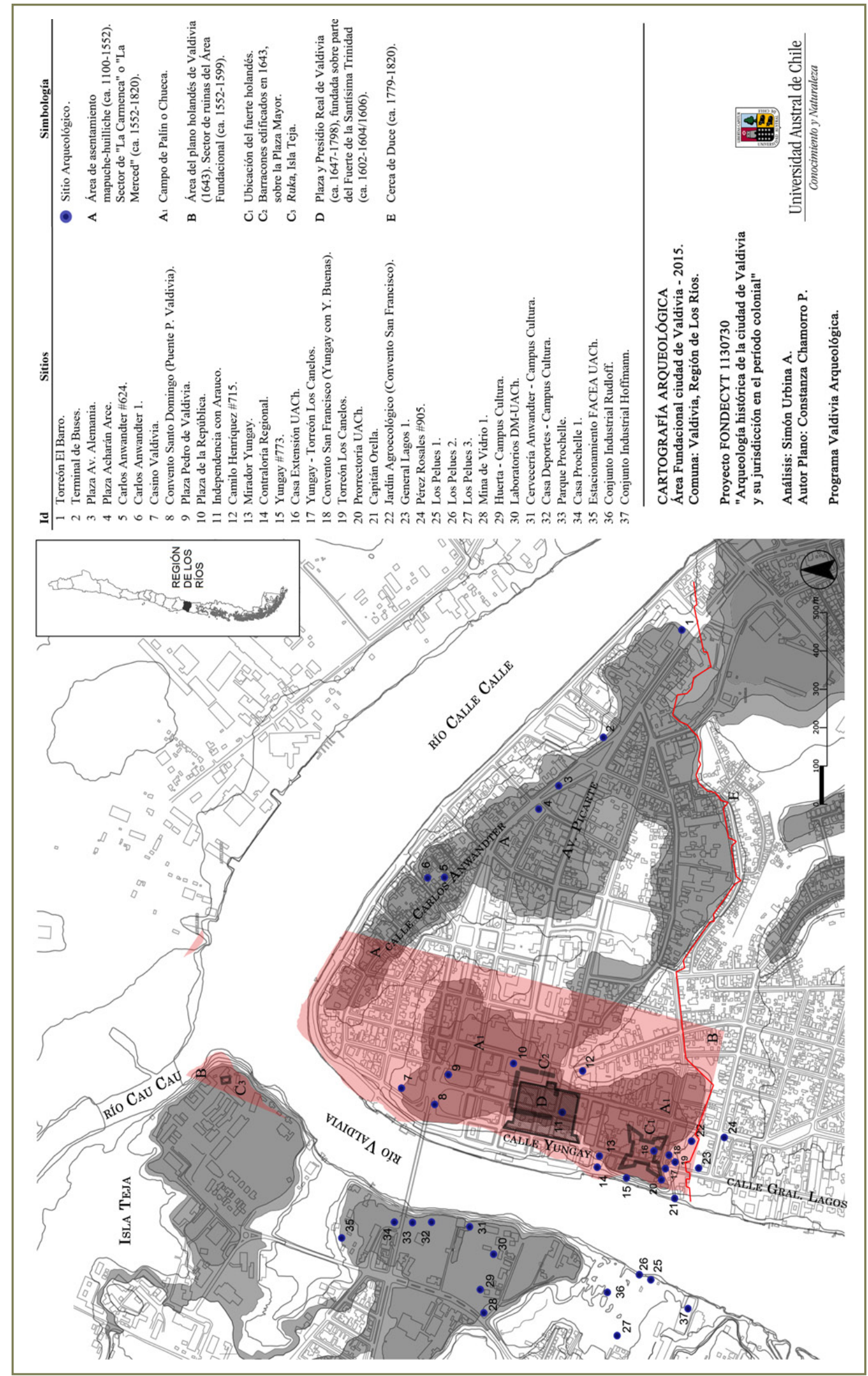

Imagen 13. Cartografía arqueológica del Área Fundacional de Valdivia 2016 (fuente: Los autores) 

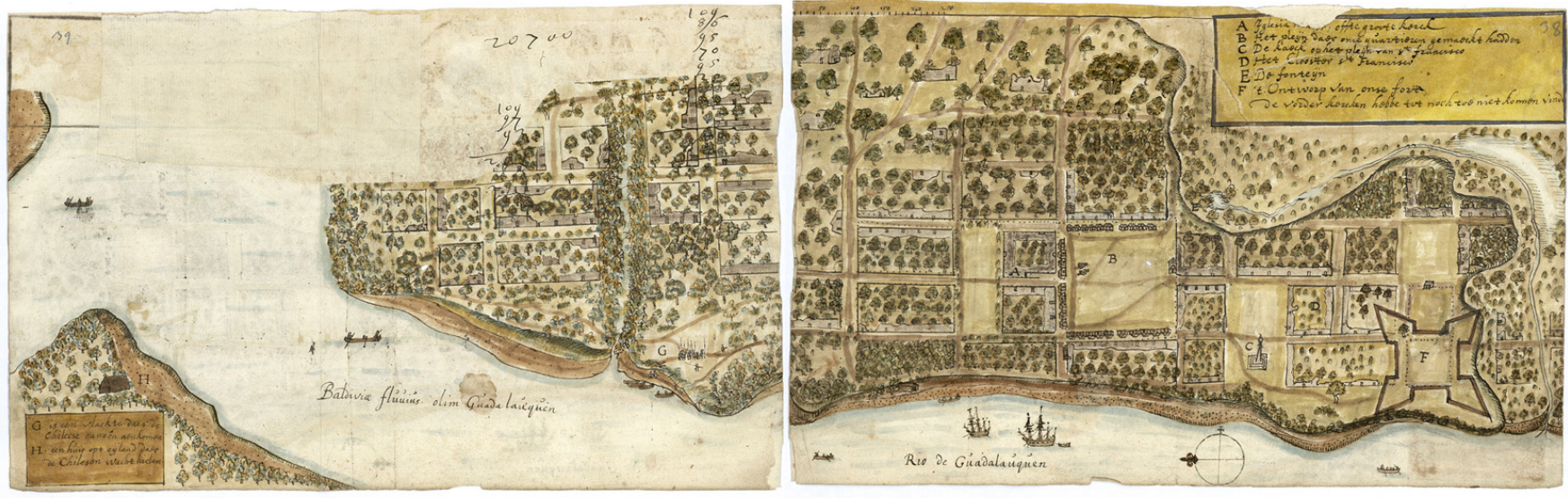

Imagen 14. Plano holandés de la ciudad de Valdivia (ca. 1643) (fuente:Universidad de Göttingen, Alemania. Gentileza de Ricardo Mendoza).

\section{ZONIFICACIÓN Y ANÁLISIS}

SECTORIAL. Con los datos disponibles,

la distribución de los sitios atribuibles al período colonial (s. XVI-XIX) que registran elementos muebles (como cerámica indígena e hispana) y/o elementos constructivos (como tejas curvas coloniales y ladrillos) aparece concentrada en las mesetas altas no inundables (28 de 37 sitios), a ambos costados del río Valdivia y en la ribera sur del río Calle-Calle.

Excepciones destacables a este patrón son los sitios Contraloría Regional, Jardín Agroecológico, Los Pelúes 1 (Isla Teja), Capitán Orella y General Lagos 1, los cuales se ubican bajo los 5 msnm e incluso bajo el nivel freático actual, en el inicio de los 'Barrios Bajos'

En el caso de Isla de Valenzuela o Teja se registran 13 sitios arqueológicos distribuidos en sectores bajos ( 6 msnm) y altos (> 14 msnm) frente al centro de Valdivia. Las evidencias cerámicas indígenas e hispanas indican que este sector de la isla es parte del 'área fundacional' de la ciudad (Urbina et al. 2012) y presenta una intensa ocupación durante el período Republicano, debido al auge industrial. Esta hipótesis concuerda parcialmente con las dataciones obtenidas para este sector insular de la ciudad, las cuales oscilan entre 1635 y 1895 DC (Tabla 2).
Los vestigios de arquitectura corresponden a puntos que registran fragmentos de tejas curvas (por ejemplo, Casa Prochelle-1, Parque Prochelle y Huerta Campus CulturalUACh) y ladrillos; a volúmenes de conjuntos industriales frente al río Valdivia (Cervecería [Museo de Arte Contemporáneo-UACh], Rudloff [en ruinas] y Hoffmann [demolida]) $y$ algunas casas insertas en dichos complejos (Anwandter [Museo Histórico UACh] y Prochelle-1 [Centro Cultural Municipal] y 2 [sede Consejo Regional Los Ríos]), donde se combinan paramentos de piedra laja, madera, ladrillo, hormigón y hierro. En las excavaciones efectuadas en las ruinas de la Cervecería Anwandter fueron recuperados fragmentos de ladrillo asociados a arquitectura en piedra laja. Las dataciones obtenidas sobre muestras de ladrillo arrojaron edades que fluctúan entre 1720, junto al río, y 1860 DC en las murallas de la rampa de acceso

El sitio General Lagos 1 -caracterizado por fragmentos de cerámica corrugada o con estriamiento anular, mayólicas polícromas, tejas y muros in situ de piedra laja y ladrillose ubica en una cota baja (< $10 \mathrm{msnm}$ ). Al apreciar el plano holandés de 1643 observamos, al sur de la fortaleza, sectores ribereños y rodeados de humedales donde se dibujan construcciones hispanas (imagen 14), las cuales aparecen en ruinas a ambos costados de un catrico o desagüe proveniente de una vertiente indicada con la letra E en el plano ${ }^{6}$.

Precisamente al sur del torreón y el convento de San Francisco, el plano de Enrique Siemsen de 1853 ubica casas con tejados en color rojo (imagen 15), dispuestas en el eje norte-sur de la calle Los Canelos, hoy General Pedro Lagos. De tal modo que esta verdadera avenida articulaba el puerto de las canoas, la Plaza Mayor y los barrios bajos del sur, probablemente poblados desde la primera etapa urbana de Valdivia (ca. 1552-1604)

Ahora bien, mientras el eje Yungay-General Lagos presenta 12 sitios arqueológicos localizados en sectores altos y bajos, en la calle Las Mercedes, hoy Carlos Anwandter, se cuentan 5 sitios, todos emplazados en sectores altos (> $12 \mathrm{msnm}$ ). Uno de ellos corresponde al torreón El Barro, que en su prolongación por calle Picarte, conocida como el antiguo camino El Barro - Cantarranas, se bifurcaba en un camino que conducía a Arique, al este, y otro que se dirigía al sur, hacia el río Angachilla y luego hacia el río Futa.

La posición topográfica de la calle Carlos Anwandter es y fue privilegiada por cuanto ocupa una loma (12-14 msnm) que corre

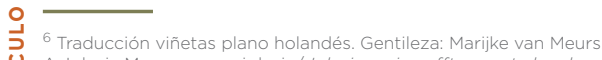

U. A. Iglesia Mayor o gran iglesia/ Iglesia maior, offte groote kerck.

B. La plaza donde hicimos nuestros cuarteles/ Hetpleÿn daer onse quartieren gemaeckt haden.

C. La iglesia en la plaza de San Francisco/ De kerck op het pleÿn van St. Francisco.

58 E. La fuente (o vertiente)/ De fonteÿn.

denuestro fuerte/ 't Ontworp van onse fort.

Las otras iglesias no las hemos encontrado hasta ahora/ De vorder kercken hebbe tot noch toe niet komen vinde

G. Es una planicie donde llegan las canoas chilenas/is een vlackte daer de Chileese canoën vorkommen H. Es una casa en la isla donde los chilenos hacen guardia/ een huis opt eÿland daer de Chileson wacht hielen.
} 
paralela al curso del río Calle-Calle y accede, por el sur, al humedal o gualve que colinda con el centro de la ciudad. Los datos arqueológicos confirman la información documental relativa a que en esta meseta, existió un caserío de rukas mapuchehuilliche ${ }^{7}$, con caminos delimitados por arboledas sembradas a mano (Mariño 1865 [1580]), a partir del cual se conformaría el barrio de Carmenga o de la Merced desde la segunda mitad del siglo XVI (imagen 12, letra A), en torno al convento homónimo (Guarda 2001). Las dataciones obtenidas en las excavaciones del sitio Carlos Anwandter-1 arrojaron fechas previas a la invasión hispana y otras más tardías que se ubican entre el año 1565 y 1895 DC (tabla 2). El sector céntrico de Valdivia, también dispuesto sobre una loma o terraza que en algunos puntos alcanza hasta 18 msnm, concentra evidencias habitacionales, funerarias, defensivas y ceremoniales, desde el período prehispánico hasta el siglo XX. Es un sector estratégico y nodal, rodeado por el nacimiento del río Valdivia - Guadalafquén y distintos humedales (imagen 14). Allí existió un extenso campo ceremonial de chueca o palin ${ }^{8}$ previo a la invasión hispana (imagen 12, letra A1), sobre el cual fue fundada en 1552 la ciudad de Valdivia -la Plaza e Iglesia Mayor al centro, el convento de Santo Domingo al norte y el de San Francisco al sur-. Se trata aproximadamente del mismo espacio donde se instalaron los barracones y se levantó el fuerte holandés en 1643, así como la Plaza Murada o Castillo de Valdivia, a partir de 1647.
En este sector destacan los sitios Casino de Valdivia, excavado entre 2007-2008, y Contraloría Regional. Este último, con $103 \mathrm{~m}^{2}$ excavados y claras evidencias estratigráficas del 'gran incendio de 1909' permitió documentar 20 fundaciones de ladrillo y piedra laja in situ correspondientes al subterráneo del edificio de La Aduana (ca. 1862-1982). Un metro bajo el sello del incendio (ca. 1909), pudieron registrarse artefactos y abundantes desechos cerámicos mapuche-huilliche, lozas británicas y cerámicas europeas, así como fragmentos de tejas curvas ${ }^{9}$. Fragmentos de tejas curvas también se encuentran superficialmente (Prorrectoría UACh) y en estratigrafía (Independencia con Arauco, Mirador Yungay, Casa Extensión-UACh y Plaza de La República), lugares donde se infiere la existencia de solares, casas o edificios hispanos.

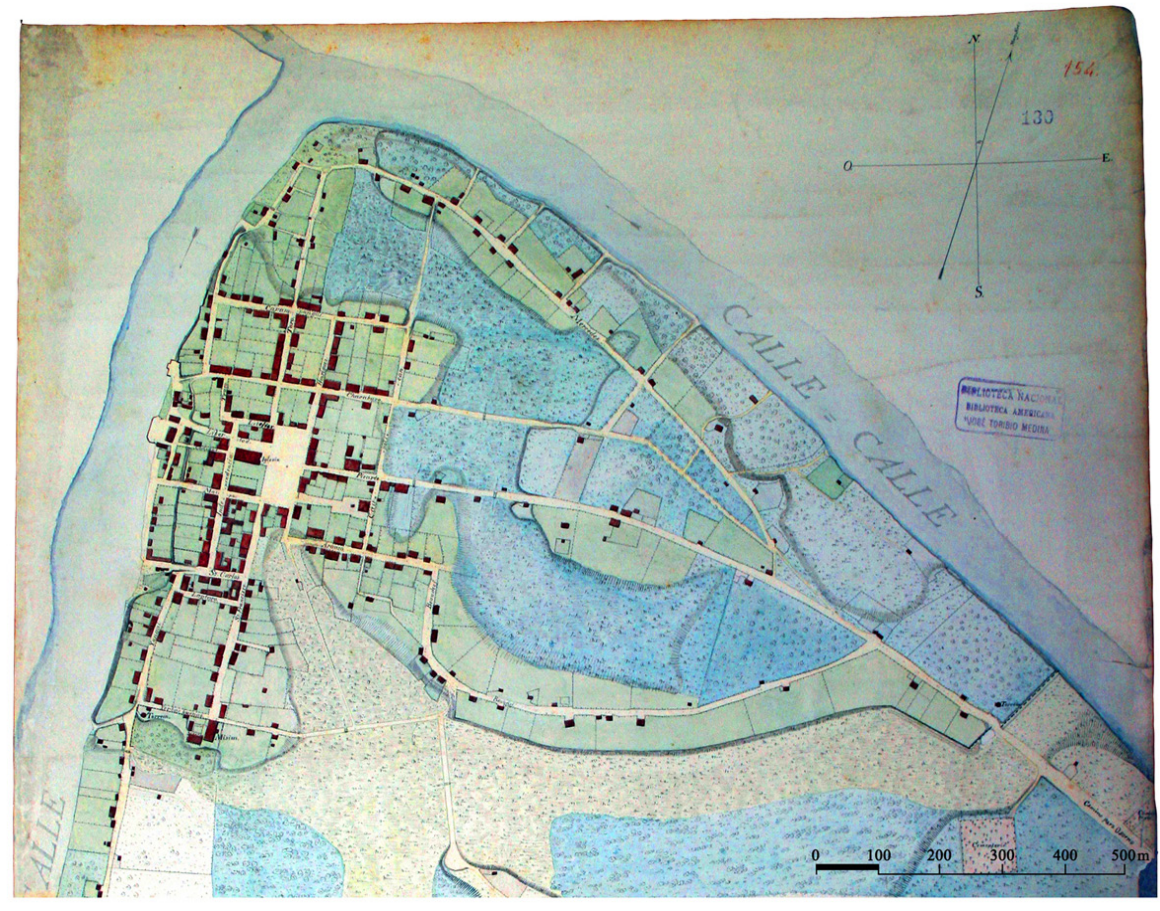

Imagen 15. Detalle plano de Valdivia de Henrique Siemsen, 1853 (fuente: Sala Medina, Biblioteca Nacional de Chile).

\footnotetext{
Población que habita desde tiempos prehispánicos el territorio ubicado entre la cuenca del río Toltén y la isla de Chiloé.

${ }^{8}$ Ritual propio y característico del pueblo Mapuche, practicado desde épocas prehispánicas en espacios de integración y congregación comunitaria tipificados en documentos coloniales como 'juntas', 'lepunes' o encuentros de palin o chueca, efectuados en campos ceremoniales o canchas. El evento ritual del palin es una actividad practicada, fundamentalmente, por hombres mapuche adultos, integrantes de una comunidad y un linaje, que participan a su vez de una red social mayor. Más que un juego, como ha sido entendido por la sociedad no mapuche, es un mecanismo que presenta delicados y profundos alcances sociales, políticos y simbólicos, constituyendo una verdadera batalla ritual. 
Cuatro dataciones de ladrillos recuperados in situ en el sitio Casino de Valdivia, fluctúan entre los años 1560-1640 DC, para el muro sur, y entre 1690-1780 DC, para el muro norte (tabla 2). Estos resultados son preliminarmente coherentes con dos ladrillos datados provenientes de las fundaciones del Castillo de Corral, con fechas que varían entre 1650 y 1715 DC (Urbina y Adán 2014). De las seis dataciones obtenidas en el sitio Contraloría Regional, una de ellas corresponde a un fragmento monócromo de Tradición Indígena datado en 1620 DC, mientras las cinco restantes se ubican entre los años 1630 y 1835 DC.

Finalmente, en la Plaza de la República de Valdivia se han excavado 49 pozos de sondeo $\left(49 \mathrm{~m}^{2}\right)$. Se han registrado 11 contextos funerarios, la mayor parte en la mitad norte (Galarce y Santander 2014), que con probabilidad se relacionan con la Iglesia Mayor del siglo XVI. Este sector se encuentra ocupado desde tiempos prehispánicos y prueba de ellos son los entierros y rellenos de hasta dos metros de profundidad con basuras domésticas y materiales constructivos, tales como bloques de piedra laja canteada, tejas curvas y planas, ladrillos de distinto tipo y baldosas de cerámica y hormigón. Se ha identificado incluso un canal de piedra laja in situ al suroeste de la plaza, sobre la calle Maipu (Galarce y Santander 2014). Cinco fechas se han obtenido en este sitio, las dos más tempranas de 1675 DC (un fragmento cerámico de Estilo Valdivia y un fragmento de teja curva), mientras los tres restantes se ubican entre 1680 y 1795 DC (Imagen 10g).
PALABRAS FINALES. El análisis de materiales constructivos es un campo poco explorado por los estudios históricos y arqueológicos en Chile, pese a la importancia que esta materia reviste para otras disciplinas como la arquitectura, la conservación, la ingeniería y la geografía histórica. El presente trabajo ha buscado construir una representación cartográfica fundamentada en un conocimiento y valoración integral de las tradiciones cerámicas -vajillas y contenedores-, en asociación al uso y descarte de ladrillos y tejas provenientes de fundaciones, pisos, muros y techumbres del período Colonial y Republicano en Valdivia, sin la cual sería imposible comprender las centenarias transformaciones económicas y sociales de sus habitantes y de los distintos sectores que conforman la urbe.

\section{REFERENCIAS}

Espinosa, P., 1859. Manual de construcciones de albañilería. Imprenta a cargo de Severiano Baz, Madrid. Galarce, P. y Santander, G., 2014. I/ Etapa sondeos arqueológicos sitio Plaza la República-Valdivia. Archeos Chile Consultores en Arqueologia. Consejo de Monumentos Nacionales.

Gordon, A., 2011. Excavación de la Residencia Fortificada de un Encomendero Español: La Casa Fuerte Santa Sylvia, Villa San Pedro, Pucón. Informe Técnico. Editado por T. Dillehay y J. Sauer. Vanderbilt University Publications in Anthropology Vol. 54. Universidad de Vanderbilt.

Guarda, G., 2009. Cuatro Siglos de evolución Urbana. Valdivia 1552-1970. Instituto de Arquitectura y Urbanismo, Universidad Austral de Chile.

Guarda, G., 2001. Nueva Historia de Valdivia. Santiago: Ediciones Universidad Católica. Mariño de Lobera, P., 1865 [1580]. Crónica del Reino de Chile. Colección de historiadores de Chile y de documentos relativos a la historia nacional (Tomo V). Santiago: Imprenta del Ferrocarril. I.
Touttsaint de Sens, M., 1860. Novísimo manual completo de arquitectura (Tomo II). Madrid: Colección de manuales de ciencia y arte.

Urbina, S. y Adán, L., 2014. "Avances en la Arqueología de Valdivia." Boletín de la Sociedad Chilena de Arqueología 43/44, 35-60

Urbina, S., Adán, L. y Chamorro, C., 2017. "Materiales constructivos y arquitectura colonial del área fundacional de Valdivia (S. XVI-XIX)." Revista de Arqueología Histórica Argentina y Latinoamericana (En prensa). Urbina, S., Adán, L., Munita, D. y Mera, R., 2012. "Arquitectura arqueológica y sitios patrimoniales sin arquitectura en el perimetro urbano de Valdivia: cartografía descriptiva actualizada y comentarios sobre su valor cientifico integral." AUS, 12, 4-9. 